\title{
Innovatively therapeutic strategy on Alzheimer's disease by daily drinking plasmon-activated water
}

\author{
C.H. Cheng ${ }^{1}$, K.J. Lin ${ }^{2}$, C.P. Yang ${ }^{1}$, Y.C. Liu ${ }^{1 *}$, C.J. Hu ${ }^{3,4}$ \\ ${ }^{1}$ Department of Biochemistry and Molecular Cell Biology, Taipei Medical University, Taipei, Taiwan \\ ${ }^{2}$ Department of Nuclear Medicine and Molecular Imaging Center, Linkou Chang Gung Memorial \\ Hospital, Tao-Yuan, Taiwan \\ ${ }^{3}$ Department of Neurology, Taipei Medical University, Taipei, Taiwan \\ ${ }^{4}$ Department of Neurology and Dementia Center, Shuang Ho Hospital, New Taipei City, Taiwan \\ *e-mail:liuyc@tmu.edu.tw
}

Key words: Alzheimer's disease, plasmon-activated water, anti-inflammation, amyloid, prevention

Motivation and Aim: Alzheimer's disease (AD) is the most common cause of dementia and $\mathrm{AD}$ is characterized by memory impairment followed by decline of many cognitive domains, including executive and visuospatial, resulting in dysfunction of activities of daily life. Amyloid accumulation in the brain which occurs before clinical presentation is the first and key step for development of AD. Many clinical trials are aiming to remove amyloid from the brain of AD patients but none is successful till now. Plasmon-activated water (PAW) is produced by gold ( $\mathrm{Au}$ ) nanoparticles (NPs) reducing the hydrogenbonded (HB) structure of water [1]. PAW has been found to contain anti-oxidation and anti-inflammation effects $[1,2]$. Here, we analyze the function of PAW in eliminating the progression of AD. The APPswe/PS1dE9 transgenic mice are treated with PAW or regular water for 9 months from 5-month-old.

Methods and Algorithms: The mice were kept in individually ventilated cages with feeding racks and water bottles were attached to the front panel of the cage which allows animals to reach food and water. Mice were maintained in a 12-hour light/ 12-hour dark cycle (12L/12D; light on 7:00, light off 19:00). The food and deionized water (DIW) or PAW were freely available and change the water every day. Six (APP/PS1) mice for each group were fed with PAW or DIW for 9 months from 5-month-old.

Results: The mice treated with PAW present the better memory performance, little amyloid and phosphorylated Tau burden in hippocampus. Collectively, our findings support the function of PAW on conferring effects of amyloid reduction and memory improvement in AD mouse model. We propose that the effect of PAW on reduction formation of senile plaque was not in synthesis of amyloid-beta protein but in prevention aggregation of amyloid-beta protein.

Conclusion: In conclusion, PAW confers the effects on reduction of amyloid accumulation and deceleration of memory decline. These new findings indicate the potential of PAW as an effective antioxidant without side effects for therapy on neuro-related diseases.

Acknowledgements: This work was supported by Taipei Medical University and Shuang Ho Hospital (105TMU-SHH-01-1).

\section{References}

1. Chen H.C. et al. (2014) Active and stable liquid water innovatively prepared using resonantly illuminated gold nanoparticles. ACS Nano. 8:2704-2713.

2. Chen H.C. et al. (2014) Innovative strategy with potential to increase hemodialysis efficiency and safety. Sci. Rep. 4:4425. 